\title{
Inflated Cost Burden of Hemodialysis during COVID-19 Pandemic
}

\author{
Xia Tan Guochun Chen Fang Yuan Yinghong Liu Hong Liu \\ Department of Nephrology, Key Lab of Kidney Disease and Blood Purification in Hunan, The Second Xiangya \\ Hospital of Central South University, Renal Research Institute of Central South University, Xiangya, China
}

Dear Editor,

The recent outbreak of coronavirus disease 2019 (COVID-19) in Wuhan, China, caused by the newly identified SARS-CoV-2 has claimed more than 80,000 people in China. This virus has posed a huge threat to the global public health and has affected $>60$ million people worldwide. Hunan Province of China declared the first level response to public health emergency from 24 January to 10 March 2020. This led to the sudden increase in the hospital burden, and hence, estimating the cost of hemodialysis facility in February 2020 of our center was crucial. We estimated that there was an increase in the cost of incenter hemodialysis facility with disinfection, protective equipment, manpower, and the COVID-19 PCR test for every patient in the center as compared to February 2019. The protective equipment included face masks for every patient, gloves, eye protection (face shields and goggles), and isolation gowns for every dialysis staff. It was estimated that the cost increased 2.57 times than that evaluated in the last year. The environmental disinfection costs were also considered which were also found to be 1.34 times higher than the previous year. Furthermore, there was twice as much health workers required to screen the patients (Fig. 1a). Overall, the human resources and the

karger@karger.com

(C) 2021 S. Karger AG, Basel

www.karger.com/bpu

Karger!
COVID-19 PCR test accounted for $89 \%$ of the total increased cost (Fig. 1b).

The human coronaviruses are transmitted mainly by the respiratory route or by contact with the infected person. As patients on hemodialysis are required to visit the hospital frequently, that is, 2-3 times a week, they are highly exposed to the virus and are susceptible to the infection. The COVID-19 outbreak in the hemodialysis center has been reported in severe infectious places [1-3]. In China, a vast number of public health interventions have been imposed to control the epidemic [4]. Due to the closure of public transport, it has created a trouble for the patients to travel to the dialysis center. However, the cost of peritoneal dialysis has not increased, giving a modest relief to the patients. The patients undergoing peritoneal dialysis have their home modality, thus not increasing risk of infection at the dialysis center. In the meantime, the training by physicians and nurses to the patients are ongoing through telephones. To summarize, the home modalities of peritoneal dialysis have overall lowered the maintenance costs during the COVID-19 pandemic. The restriction measures have not significantly affected the ESRD patients on peritoneal dialysis as compared to the patients undergoing hemodialysis at hospital facility. 


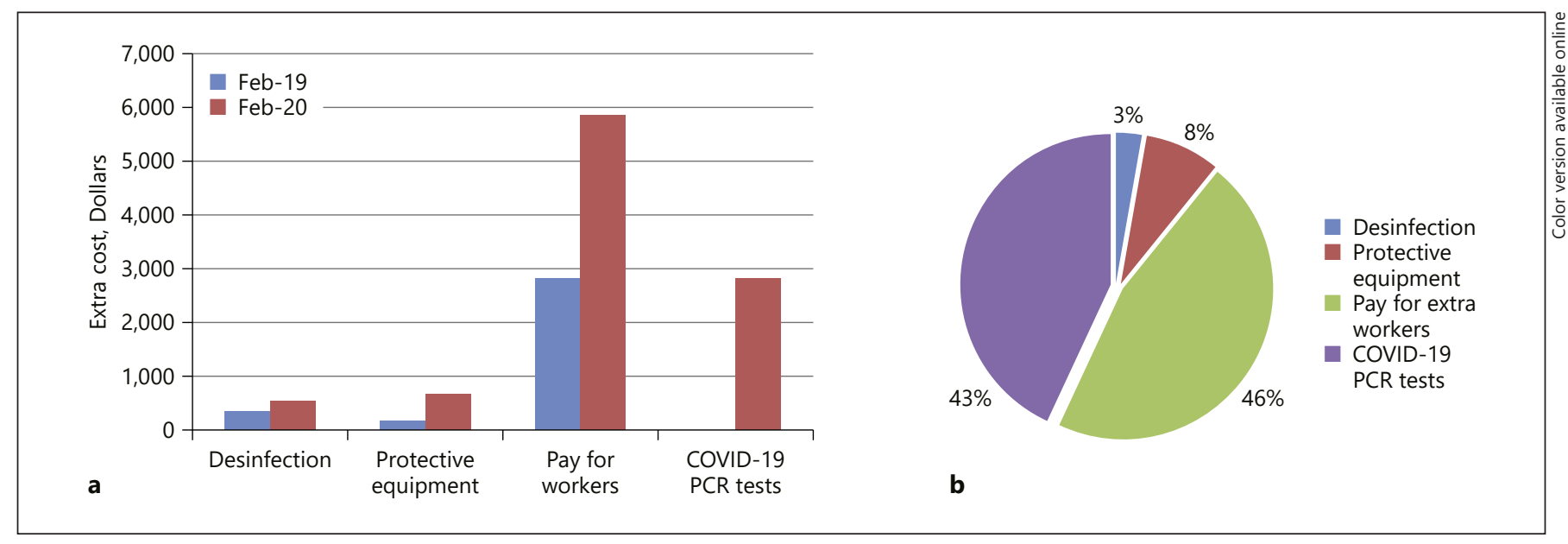

Fig. 1. a Compared to the same month of 2019, the increased cost of in-center facility hemodialysis included disinfection, protective equipment, salary for extra workers, and the COVID-19 PCR test for every patient in our center. The cost of protective equipment is 2.57 times than that of the last year. The cost of environment dis-

infection is 1.34 times than that of the previous year. Nearly twice as much workers were needed to help screen patients. b The human resources and the cost for the COVID-19 PCR test account for $89 \%$ of the increased cost. COVID-19, coronavirus disease 2019.

\section{Acknowledgement}

Funding Sources

The authors thank all nurses in our hemodialysis center for helping in manuscript preparation.

This work was supported by a research grant (81770714) from the National Natural Science Foundation of China.

\section{Conflict of Interest Statement}

The authors have no conflicts of interest to declare.

\section{Author Contributions}

Conceived and designed the experiments: Hong Liu and Xia Tan. Analyzed the data: Xia Tan, Guochun Chen, Fang Yuan, and Yinghong Liu. Wrote the paper: Xia Tan and Guochun Chen.

\section{References}

1 Ma Y, Diao B, Lv X, Zhu J, Liang W, Liu L, et al. Novel coronavirus disease in hemodialysis (HD) patients: report from one HD center in Wuhan, China. 2019.

2 Rombolà G, Heidempergher M, Pedrini L, Farina $\mathrm{M}$, Aucella F, Messa $\mathrm{P}$, et al. Practical indications for the prevention and management of SARS-CoV-2 in ambulatory dialysis patients:lessons from the first phase of the epidemics in Lombardy. J Nephrol. 2020 Apr; 33(2):193-6.
3 Xiong F, Tang H, Liu L, Tu C, Tian JB, Lei CT, et al. Clinical characteristics of and medical interventions for COVID-19 in hemodialysis patients in Wuhan, China. J Am Soc Nephrol. 2020 Jul;31(7):1387-97.

4 Lin Q, Zhao S, Gao D, Lou Y, Yang S, Musa SS, et al. A conceptual model for the coronavirus disease 2019 (COVID-19) outbreak in Wuhan, China with individual reaction and governmental action. Int J Infect Dis. 2020;93 211-6. 